Journal of Zhejiang University-SCIENCE C (Computers \& Electronics)

ISSN 1869-1951 (Print); ISSN 1869-196X (Online)

www.zju.edu.cn/jzus; www.springerlink.com

E-mail: jzus@zju.edu.cn

\title{
Editorial:
}

\section{Brain-machine interface (BMI) and cyborg intelligence}

\section{Zhao-hui WU}

\author{
Guest Editor-in-Chief for this special issue \\ Zhejiang University, Hangzhou 310027, China \\ E-mail:wzh@zju.edu.cn
}

doi: $10.1631 /$ jzus.C1400325

Brain-machine interfaces (BMIs) aim at building a direct communication pathway between the brain and an external device, and represent an area of research where significant progress has been made during the past decade. Based on BMIs, mind information can be read out by neural signals to control machine actuators, and machine-coded sensory information can be delivered to specific areas in the brain. BMI techniques provide the opportunity to integrate machine intelligence with biological intelligence at multiple levels to develop a more powerful intelligent system, thereby creating a new field called 'cyborg intelligence'.

Traditional artificial intelligence (AI) involves learning how to emulate human-like intelligence and the creation of machines or software systems that exhibit intelligence. Successful AI includes natural language processing, speech recognition, smart search engines, face recognition, and Q\&A systems. Although AI techniques show advantages in highperformance computation, probabilistic models, statistical reasoning, optimization, and almost unlimited storage, modern AI systems are unlikely to match humans in learning, high-level reasoning, and flexible adaptation to varying environments, which are recognized as the best features of biological intelligence. Because of their obvious complementary strengths, studies of the convergence of machine and biological intelligence, i.e., cyborg intelligence, are of great

(C) Zhejiang University and Springer-Verlag Berlin Heidelberg 2014 significance in maximizing their capabilities through their integration.

The central problems of cyborg intelligence include information fusion and representation in sensory-motor integration, cognitive computational models in brain-machine collaborations, statistical models for decoding and encoding brain signals, computational models and architecture for cyborg intelligence, and related data and computation standards. To explore this exciting new field, two workshops were held in Changsha and Hangzhou, China, in October and December 2013, respectively. More than 30 experts from the neuroscience, neuroengineering, and computer science fields came together to discuss scientific problems and research trends in the cyborg intelligence area. This special issue includes five of the most interesting and significant presentations describing state-of-the-art research in this field.

How can we better understand biological intelligence? Chen et al. (2014) addressed a biological intelligence problem in neuroscience, i.e., what are the details of the process of eye-specific segregation in the dorsal lateral geniculate nucleus (dLGN) and superior colliculus (SC) in postnatal mice. The study showed that the development of eye-specific segregation in the $\mathrm{SC}$ is completed before postnatal day 12 (P12), which is earlier than in the dLGN (P20).

How can we enhance intelligence in perception and motor functionality reconstruction? Zhou et al. (2014) reported a study about the nature of physiological functional recovery of reinnervated muscles using a new neural-function reconstruction method, targeted muscle reinnervation (TMR). The results showed that the active myoelectric signal reconstructed in the targeted muscle could be acquired one week after TMR surgery, and its amplitude gradually became stronger over time. The findings will help us fine tune the TMR procedure to maximize its capability for improving the control of myoelectric multifunctional prostheses. 
How can we decode brain signals relevant to music? Wu et al. (2014) proposed a scale-free brainwave music (SFBM) technique to listen to brain activity as a piece of music, and involved translating a scalp EEG into musical notes according to the power law of both the EEG and music. The brain ensemble consonance was modulated by EEG phase synchronization. This novel method is a new attempt to express an EEG in an auditory and musical form, and can be used for EEG monitoring and bio-feedback.

How can we connect a BMI system to a speech recognition system? Wang et al. (2014) presented an intelligent wheelchair, controlled by a coordinated control mechanism based on a brain-computer interface (BCI) and speech recognition. By performing appropriate speech activities, users can navigate the wheelchair using four steering behaviors (start, stop, turn left, and turn right). The experimental results demonstrated the efficiency of the coordinated control mechanism with satisfactory path and time optimality ratios, and showed that speech recognition is a fast and accurate supplement for BCI-based control systems.

How can we build a cyborg intelligence system for medical use? Qi et al. (2014) presented a new BCI-based responsive stimulation system for use in epilepsy monitoring. With an efficient morphologybased seizure detector, seizure events which trigger electronic stimulations to the cortex of the brain can be identified in the early stages. The results showed that most seizures could be detected successfully with a short average time delay, and be suppressed within $10 \mathrm{~s}$. The system can be used to control epileptic seizures effectively and has potential in clinical applications.

Related and valuable future subfields of cyborg intelligence include neuromorphic computing, in which electronic analog circuits are designed to mimick neuro-biological architectures present in the nervous system, animal robots, which build animal cyborgs to take advantage of the strengths of both machines and animals, and BMI-based rehabilitation devices.

We would like to express our appreciation to the distinguished reviewers, whose expertise and professionalism have contributed significantly to the quality of this special issue. We would also like to thank Helen (Y.H.) ZHANG, Managing Editor of Journals of Zhejiang University-SCIENCE $(A / B / C)$, and Zi-yang ZHAI, editor of this special issue, for their helpful guidance and constructive feedback during the entire process of organizing and publishing this special issue.

\section{References}

Chen, Y.Q., Diao, Y.P., Duan, J.G., et al., 2014. Timedependent changes in eye-specific segregation in the dorsal lateral geniculate nucleus and superior colliculus of postnatal mice. J. Zhejiang Univ.-Sci. C (Comput. \& Electron.), 15(10):807-812. [doi:10.1631/jzus.C1400 153]

Qi, Y., Ma, F.Q., Ge, T.T., et al., 2014. A bidirectional brain computer interface for effective epilepsy control. $J$. Zhejiang Univ.-Sci. C (Comput. \& Electron.), 15(10): 839-847. [doi:10.1631/jzus.C1400152]

Wang, H.T., Li, Y.Q., Yu, T.Y., 2014. Coordinated control of an intelligent wheelchair based on a brain-computer interface and speech recognition. J. Zhejiang Univ.-Sci. C (Comput. \& Electron.), 15(10):832-838. [doi:10.1631/ jzus.C1400150]

Wu, D., Li, C.Y., Liu, J., et al., 2014. Scale-free brain ensemble modulated by phase synchronization. J. Zhejiang Univ.-Sci. C (Comput. \& Electron.), 15(10):821-831. [doi:10.1631/jzus.C1400199]

Zhou, H., Yang, L., Wu, F.X., et al., 2014. Exploring the mechanism of neural-function reconstruction by reinnervated nerves in targeted muscles. J. Zhejiang Univ.-Sci. C (Comput. \& Electron.), 15(10):813-820. [doi:10.1631/ jzus.C1400154] 